\title{
FUNCTIONAL EQUATION AND ZEROS ON THE CRITICAL LINE OF THE QUADRILATERAL ZETA FUNCTION
}

\author{
TAKASHI NAKAMURA
}

\begin{abstract}
For $0<a \leq 1 / 2$, we define the quadrilateral zeta function $Q(s, a)$ using the Hurwitz and periodic zeta functions and show that $Q(s, a)$ satisfies Riemann's functional equation studied by Hamburger, Heck and Knopp. Moreover, we prove that for any $0<a \leq 1 / 2$, there exist positive constants $A(a)$ and $T_{0}(a)$ such that the number of zeros of the quadrilateral zeta function $Q(s, a)$ on the line segment from $1 / 2$ to $1 / 2+i T$ is greater than $A(a) T$ whenever $T \geq T_{0}(a)$.
\end{abstract}

\section{Contents}

1. Introduction and Statement of the Main Results 1

1.1. Main results 1

1.2. Zeros of zeta functions on the critical line 2

1.3. Hamburger's, Hecke's and Knopp's Theorems 4

1.4. Variations of Knopp's Theorem 5

2. Proofs 7

2.1. Functional equation and integral representation 7

2.2. Lemmas 9

2.3. Proof of the existence of zeros on the critical line 12

$\begin{array}{ll}\text { Acknowledgments } & 17\end{array}$

$\begin{array}{ll}\text { References } & 18\end{array}$

\section{Introduction and Statement of the Main Results}

1.1. Main results. For $0<a \leq 1$, define the Hurwitz zeta function $\zeta(s, a)$ by

$$
\zeta(s, a):=\sum_{n=0}^{\infty} \frac{1}{(n+a)^{s}}, \quad \sigma>1,
$$

and the periodic zeta function $\operatorname{Li}(s, a)$ by

$$
\operatorname{Li}(s, a):=\sum_{n=1}^{\infty} \frac{e^{2 \pi i n a}}{n^{s}}, \quad \sigma>1 .
$$

The Dirichlet series of $\zeta(s, a)$ and $\operatorname{Li}(s, a)$ converge absolutely in the half-plane $\sigma>1$ and uniformly in each compact subset of this half-plane. Moreover, the Hurwitz zeta function has analytic continuation to $\mathbb{C}$ except $s=1$, where there is a simple pole with residue

2010 Mathematics Subject Classification. Primary 11M35, Secondary 11M26.

Key words and phrases. Functional equation, converse theorems, Quadrilateral zeta function, Zeros on the critical line. 
1 (e.g., [1, Chapter 12]). In contrast, the Dirichlet series of the function $\operatorname{Li}(s, a)$ with $0<a<1$ converges uniformly in each compact subset of the half-plane $\sigma>0$ (e.g., [15, p. 20]). Furthermore, the function $\operatorname{Li}(s, a)$ with $0<a<1$ is analytically continuable to the whole complex plane (e.g., [15, Chapter 2.2]). We clearly have $\zeta(s, 1)=\operatorname{Li}(s, 1)=\zeta(s)$, where $\zeta(s)$ is the Riemann zeta function.

For $0<a \leq 1 / 2$, we define the quadrilateral zeta function $Q(s, a)$ as

$$
2 Q(s, a):=\zeta(s, a)+\zeta(s, 1-a)+\operatorname{Li}(s, a)+\operatorname{Li}(s, 1-a) .
$$

Based on the facts mentioned above, the function $Q(s, a)$ can be continued analytically to the whole complex plane except $s=1$. The first main theorem is the following functional equation of the quadrilateral zeta function $Q(s, a)$.

Theorem 1.1. For $0<a \leq 1 / 2$, it holds that

$$
Q(1-s, a)=\frac{2 \Gamma(s)}{(2 \pi)^{s}} \cos \left(\frac{\pi s}{2}\right) Q(s, a) .
$$

Moreover, we show the following, which implies that $Q(s, a)$ has infinitely many zeros on the critical line $\sigma=1 / 2$.

Theorem 1.2. For any $0<a \leq 1 / 2$, there exist positive constants $A(a)$ and $T_{0}(a)$ such that the number of zeros of $Q(s, a)$ on the line segment from $1 / 2$ to $1 / 2+i T$ is greater than $A(a) T$ whenever $T \geq T_{0}(a)$.

We share some remarks on the functional equation and zeros on the critical line of zeta functions in the next three subsections. Note that the quadrilateral zeta function $Q(s, a)$ also has the following remarkable properties. From $[17,(2.4)]$, it holds that

$$
Q(0, a)=-1 / 2=\zeta(0) \text { for all } 0<a \leq 1 / 2 .
$$

For $n \in \mathbb{N}$, it is shown in [18, Corollary 3.7] that $Q(-n, a)$ and $\pi^{-2 n} Q(2 n, a)$ are rational functions of $e^{2 \pi i a}$ with rational coefficients. By (1.2), the function $Q(s, a)$ has simple zeros at the negative even integers. Furthermore, it is proved in [17, Theorem 1.1] that there exists $a_{0}=0.1183751396 \ldots$ such that

(1) $Q\left(\sigma, a_{0}\right)$ has a unique double real zero at $\sigma=1 / 2$ when $\sigma \in(0,1)$,

(2) for any $a \in\left(a_{0}, 1 / 2\right]$, the function $Q(\sigma, a)$ has no real zero in $\sigma \in(0,1)$,

(3) for any $a \in\left(0, a_{0}\right), Q(\sigma, a)$ has at least two real zeros in $\sigma \in(0,1)$.

In addition, for $0<a \leq 1 / 2$, it is shown in [17, Proposition 1.5] that

$$
N(T, Q(s, a))=\frac{T}{\pi} \log T-\frac{T}{\pi} \log \left(2 e \pi a^{2}\right)+O_{a}(\log T),
$$

where $N(T, F)$ is the number of non-real zeros of a function $F(s)$ with $|\Im(s)|<T$ when $T$ is sufficiently large. Moreover, we prove in [17, Proposition 1.4] that $Q(s, a)$ has infinitely many complex zeros in the region of absolute convergence and the critical strip when $a \in \mathbb{Q} \cap(0,1 / 2) \backslash\{1 / 6,1 / 4,1 / 3\}$.

1.2. Zeros of zeta functions on the critical line. The famous Riemann hypothesis asserts that the real part of every non-real zero of the Riemann zeta function is $1 / 2$. The study to establish the lower bound for the number of zeros of $\zeta(s)$ on the critical line $\sigma=1 / 2$ has long history. Denote by $N_{\mathrm{Ri}}(T)$ the number of zeros $\rho=\beta+i \gamma$ of the Riemann zeta function $\zeta(s)$ with $\beta=1 / 2$ and $0<\gamma \leq T$. In 1914, Hardy proved that 
$N_{\mathrm{Ri}}(T) \rightarrow \infty$ if $T \rightarrow \infty$. Later, Hardy and Littlewood [9] showed the following (see also [5, Chapter 11.2] and [22, Chapter 10.7]):

Theorem A (Hardy and Littlewood [9, Theorem A]). There are constants $A>0$ and $T_{0}>0$ such that $N_{\mathrm{Ri}}(T) \geq A T$ whenever $T>T_{0}$.

In 1942, Selberg proved that there exists $A>0$ such that

$$
N_{\mathrm{Ri}}(T) \geq A T \log T \text {. }
$$

Note that the numerical value of the constant $A$ in Selberg's theorem was very small. However, Levinson [16] greatly improved Selberg's result and showed that $A \geq 1 / 3$. Furthermore, Conrey [4] proved that $A \geq 0.4088$. The current (June 2021) best result, which was proved by Kühn, Robles, and Zeindler [13], for the lower bound of $A$ is

$$
A \geq 0.410725 \text {. }
$$

It is well-known that the Riemann zeta function $\zeta(s)$ does not vanish in the region of absolute convergence by the Euler product. Next, we review some facts about the zeros on the vertical line $\sigma=1 / 2$ of the Epstein and Hurwitz zeta functions, which have complex zeros in the half-plane $\sigma>1 / 2$ (e.g., [11, Chapter 7.4.3] and [15, Chapter 8.4]).

Let $B(x, y)=a x^{2}+b x y+c y^{2}$ be a positive definite integral binary quadratic form, and denote by $r_{B}(n)$ the number of solutions of the equation $B(x, y)=n$ in integers $x$ and $y$. Then, the Epstein zeta function for the form $B$ is defined by the ordinary Dirichlet series

$$
\zeta_{B}(s):=\sum_{(x, y) \in \mathbb{Z}^{2} \backslash(0,0)} \frac{1}{B(x, y)^{s}}=\sum_{n=1}^{\infty} \frac{r_{B}(n)}{n^{s}}
$$

for $\sigma>1$. It is widely known that the function $\zeta_{B}(s)$ admits analytic continuation into the entire complex plane except for a simple pole at $s=1$ with residue $2 \pi \Delta^{-1}$, where $\Delta:=\sqrt{4 a c-b^{2}}$ (e.g., [6, Section 1]). Moreover, the function $\zeta_{B}(s)$ satisfies the functional equation

$$
\left(\frac{\Delta}{2 \pi}\right)^{s} \Gamma(s) \zeta_{B}(s)=\left(\frac{\Delta}{2 \pi}\right)^{1-s} \Gamma(1-s) \zeta_{B}(1-s) .
$$

Denote by $N_{\mathrm{Ep}}(T)$ the number of zeros of the Epstein zeta function $\zeta_{B}(s)$ on the critical line and whose imaginary part is smaller than $T>0$. In 1935, Potter and Titchmarsh [19] showed that $N_{\mathrm{Ep}}(T) \gg T^{1 / 2-\varepsilon}$. Subsequently, Sankaranarayanan [20] obtained $N_{\mathrm{Ep}}(T) \gg$ $T^{1 / 2} / \log T$, and Jutila and Srinivas [14] proved that $N_{\text {Ep }}(T) \gg T^{5 / 11-\varepsilon}$. As the current (June, 2021) best result, Baier, Srinivas, and Sangale [2] showed that

$$
N_{\mathrm{Ep}}(T) \gg T^{4 / 7-\varepsilon} \text {. }
$$

A key to the proof of the estimation $N_{\mathrm{Ep}}(T) \gg T^{4 / 7-\varepsilon}$ shown in [2] is the first power mean of an ordinary Dirichlet series $\sum_{n=1}^{\infty} b_{n} n^{-s}$ with $b_{n} \in \mathbb{C}$ satisfying certain conditions cannot be too small. By zeros on the critical line and the functional equation of the Epstein zeta function $\zeta_{B}(s)$ mentioned above, the quadrilateral zeta function $Q(s, a)$ has many analytical properties in common with the Epstein zeta function (and the Riemann zeta function). It should be mentioned that the gamma factor of $Q(s, a)$ does not depend on the parameter $0<a<1 / 2$ from Theorem 1.1, but the gamma factor of $\zeta_{B}(s)$ depends on the discriminant $\Delta$ of the positive definite integral binary quadratic form $B(x, y)$. Furthermore, we can see that the lower bound for the zeros of $Q(s, a)$ on the critical line is better than that of $\zeta_{B}(s)$ by virtue of Theorem 1.2 at present. 
For the Hurwitz zeta function $\zeta(s, a)$ with $a=1 / 3,2 / 3,1 / 4,3 / 4,1 / 6$, or $5 / 6$, Gonek [7] showed that there exists a constant $0<c<1$ such that the number of zeros (including multiplicities) of $\zeta(s, a)$ on the segment $(1 / 2,1 / 2+i T)$ is $\leq(c+o(1))(T / 2 \pi) \log T$ as $T$ tends to infinity. Moreover, he concluded with the following conjecture:

Conjecture (Gonek [7]). If $0<a<1$ is rational and $a \neq 1 / 2$, then the Hurwitz zeta function $\zeta(s, a)$ has $\ll T$ zeros on the segment $(1 / 2,1 / 2+i T)$.

Based on this conjecture and the facts above, we can guess that proving the existence of $\gg T$ zeros on the line segment $(1 / 2,1 / 2+i T)$ of the Hurwitz or Epstein zeta functions is difficult because these zeta functions have no Euler product in general. However, we show that the quadrilateral zeta function $Q(s, a)$ has $\gg T$ zeros on the line segment $(1 / 2,1 / 2+i T)$ even though $Q(s, a)$ cannot be written as an Euler product (see (1.1)).

Remark. The quadrilateral zeta function $Q(s, a)$ with $a \in \mathbb{Q}$ can be essentially expressed as a linear combination of Euler products from (1.6). Hence, under the GRH and some assumptions on well-spacing of zeros for Dirichlet $L$-functions, we could show that $Q(s, a)$ with $a \in \mathbb{Q} \cap(0,1 / 2) \backslash\{1 / 3,1 / 4,1 / 6\}$ have $100 \%$ of zeros on the line $\sigma=1 / 2$ if we could replace the function $\sum_{j=1}^{N} b_{j} L\left(s, \chi_{j}\right)$, where $b_{j} \in \mathbb{R} \backslash\{0\}$, in Bombieri and Hejhal $\left[3\right.$, Theorem A] by the function $\sum_{j=1}^{N}\left(\beta_{1 j}+\beta_{2 j} q^{s}\right) L\left(s, \chi_{j}\right)$, where $\beta_{1 j}, \beta_{2 j} \in \mathbb{C} \backslash\{0\}$ and $q$ is a natural number. However, it seems to be extremely difficult for us to relax their assumptions in $\left[3\right.$, Theorem A] as above (even when $\beta_{2 j}=0$ ). It is worth noting that $Q(s, a)$ with $a \in \mathbb{R} \backslash \mathbb{Q}$ can be expressed as neither an ordinary Dirichlet series nor a linear combination of Euler products. Despite these facts, we can prove Theorem 1.2 by modifying the proof of Hardy and Littlewood's classical ideas in [9, Sections 2, 3, and 4] (see also [5, Chapter 11.2] and [22, Chapter 10.7]).

1.3. Hamburger's, Hecke's and Knopp's Theorems. It is widely known that $\zeta(s)$ satisfies the functional equation

$$
\zeta(1-s)=\frac{2 \Gamma(s)}{(2 \pi)^{s}} \cos \left(\frac{\pi s}{2}\right) \zeta(s) .
$$

As the first converse theorem for the Riemann zeta function $\zeta(s)$, Hamburger [8] proved that $\zeta(s)$ is characterized by the functional equation (1.3) (see also Siegel [21] and Titchmarsh [22, Chapter 2.13]).

Theorem B (Hamburger [8, Satz 1]). Let $G(s)$ be an entire function of finite order, $P(s)$ be a polynomial, and suppose that

$$
f(s):=\frac{G(s)}{P(s)}=\sum_{n=1}^{\infty} \frac{a(n)}{n^{s}}, \quad a(n) \in \mathbb{C},
$$

the series being absolutely convergent for $\sigma>1$. Assume that

$$
\pi^{-s / 2} \Gamma\left(\frac{s}{2}\right) f(s)=\pi^{-(1-s) / 2} \Gamma\left(\frac{1-s}{2}\right) f(1-s) .
$$

Then, we have $f(s)=C \zeta(s)$, where $C$ is a constant.

Hecke [10, Section 1] showed that Hamburger's Theorem can be rewritten as: the following three conditions characterize $\zeta(2 s)$ up to a constant factor. 
-(1)- The function $\phi(s)$ is meromorphic, and $P(s) \phi(s)$ is an entire function of finite genus with a suitable polynomial $P(s)$.

-(2)- The function $R(s)=\pi^{-s} \Gamma(s) \phi(s)$ satisfies the functional equation $R(s)=R(1 / 2-s)$.

-(3a)- Both functions $\phi(s)$ and $\phi(s / 2)$ can be expanded in a Dirichlet series that converges in a half-plane.

Moreover, Hecke [10] proved that the expressibility of $\phi(s / 2)$ as a Dirichlet series in -(3a)- can be replaced by the following restriction on the poles of $\phi(s)$. More precisely, he showed that $\zeta(2 s)$ (up to a constant factor) is uniquely determined by -(1)-, - (2)-, and

-(3b)- The function $\phi(s)$ can be expanded in a Dirichlet series that converges somewhere and the only pole allowed for $\phi(s / 2)$ is $s=1$.

It is quite natural to relax the conditions introduced by Hecke. Knopp [12] showed the following, which implies that there are infinitely many linearly independent solutions if we drop the pole condition -(3b)- above by using the Riemann-Hecke correspondence between ordinary Dirichlet series with functional equations and modular forms or the generalized Poincaré series.

Theorem C (Knopp [12, Theorem 1]). Let $\sigma_{0} \geq 1 / 4$ and $\mathcal{A}\left(\sigma_{0}\right)$ be the space of all rational functions $A(s)$ with poles restricted to the vertical strip $1 / 2-\sigma_{0} \leq \Re(s) \leq \sigma_{0}$ and satisfying $A(1 / 2-s)=A(s)$. Let $A_{H}\left(\sigma_{0}\right)$ be the subspace of $A$ in $A\left(\sigma_{0}\right)$ such that $R(s)-A(s)$ is entire for some $R(s)=\pi^{-s} \Gamma(s) \phi(s)$ satisfying -(1)-, -(2)-, and -(3)- The function $\phi(s)$ can be expanded in a Dirichlet series that converges somewhere. Then with $\mathbb{N} \ni n>\left[\sigma_{0} / 2+3 / 8\right]+2$ and $A_{1}, \ldots, A_{n} \in A\left(\sigma_{0}\right)$, some nontrivial linear combination of the $A_{j}$ is in $A_{H}\left(\sigma_{0}\right)$.

According to the theorems by Hamburger, Hecke, and Knopp, we can see that the conditions to characterize $\zeta(s)$ introduced by Hamburger or Hecke are so polished that a slight weakening of their conditions leads to infinitely many counterexamples, as mentioned by Knopp. Note that Knopp's theorem does not provide any explicit representation for the coefficients of $a(n)$ of the Dirichlet series satisfying condition -(3)-. However, as analogues or improvements to Knopp's Theorem, in the next subsection, we show that the zeta function $Q(s, a)$ defined explicitly in Section 1.1 fulfills the assumption -(2)- and some modified conditions of -(1)- and -(3a)- or -(3b)-.

1.4. Variations of Knopp's Theorem. Now, we consider some variations of Knopp's Theorems, namely, we properly modify conditions -(1)-, -(3a)-, and -(3b)- introduced by Hecke and prove that $Q(s, a)$ fulfills the reshaped conditions.

First, we have the following immediately from Theorem 1.1.

Corollary 1.3. The function $Q(2 s, a)$ satisfies -(1)-, -(2)- and -(3a')- Both functions $\phi(s)$ and $\phi(s / 2)$ can be expanded in a GENERAL Dirichlet series that converges in a half-plane.

Next, let $\varphi$ be the Euler totient function and $\chi$ be a primitive Dirichlet character of the conductor of $q$. Let $L(s, \chi):=\sum_{n=1}^{\infty} \chi(n) n^{-s}$ be the Dirichlet $L$-function. Then, for $0<r<q$, where $q$ and $r$ are relatively prime integers, we have

$$
\zeta(s, r / q)=\sum_{n=0}^{\infty} \frac{1}{(n+r / q)^{s}}=\sum_{n=0}^{\infty} \frac{q^{s}}{(r+q n)^{s}}=\frac{q^{s}}{\varphi(q)} \sum_{\chi \bmod q} \bar{\chi}(r) L(s, \chi) .
$$


In addition, let $G(r, \bar{\chi})$ denote the (generalized) Gauss sum $G(r, \bar{\chi}):=\sum_{n=1}^{q} \bar{\chi}(n) e^{2 \pi i r n / q}$ associated with a Dirichlet character $\bar{\chi}$. Then we have

$$
\operatorname{Li}(s, r / q)=q^{-s} \sum_{n=1}^{q} e^{2 \pi i r n / q} \zeta(s, n / q)=\frac{1}{\varphi(q)} \sum_{\chi \bmod q} G(r, \bar{\chi}) L(s, \chi) .
$$

Hence, from (1.4) and (1.5), it holds that

$$
Q(s, r / q)=\frac{1}{2 \varphi(q)} \sum_{\chi \bmod q}(1+\chi(-1))\left(\bar{\chi}(r) q^{s}+G(r, \bar{\chi})\right) L(s, \chi) .
$$

Therefore, we have the following from the functional equation (1.2).

Corollary 1.4. The function $Q(2 s, r / q)$ satisfies -(1)-, -(2)-, and -(3b')- There exists a positive integer $q$ such that $q^{-2 s} \phi(s)$ can be expanded in a Dirichlet series that converges somewhere, and the only pole allowed for $\phi(s / 2)$ is $s=1$.

For $q \in \mathbb{N}$, put $H(s, q):=\left(q^{s}+q^{1-s}\right)^{-1}$. Then, we can see that $H(s, q)=H(1-s, q)$, and $q^{s} H(s, q)$ is written as an ordinary Dirichlet series by

$$
q^{s} H(s, q)=\frac{q^{s}}{q^{s}+q^{1-s}}=\frac{1}{1+q^{1-2 s}}=\sum_{k=0}^{\infty} \frac{(-q)^{k}}{q^{2 k s}}, \quad \sigma>1 / 2 .
$$

From (1.6), the function $q^{-s} Q(s, r / q)$ can be expressed as an ordinary Dirichlet series. Therefore, we can see that

$$
H(s, q) Q(s, r / q)=q^{s} H(s, q) \cdot q^{-s} Q(s, r / q)
$$

is also written by an ordinary Dirichlet series. Moreover, the function

$$
\left(1-q^{1-s}\right)\left(1+q^{1-2 s}\right) H(s, q) Q(s, r / q)
$$

is entire. Hence, we have the following from Theorem 1.1.

Corollary 1.5. The function $H(2 s, q) Q(2 s, r / q)$ satisfies (2), (3a), and -(1')- The function $\phi(s)$ is meromorphic and $D(s) \phi(s)$ is an entire function of finite genus with a suitable DIRICHLET polynomial $D(s)$.

Corollaries 1.3, 1.4 and 1.5 can be regarded as analogues of Theorem C. Note that the functions appearing in Knopp's theorem have poles in the strip $1 / 2-\sigma_{0} \leq \Re(s) \leq \sigma_{0}$, where $\sigma_{0} \geq 1 / 4$, from the condition -(3)-, but the zeta function $Q(2 s, a)$ has only one pole at $s=1 / 2$ (see condition -(3b)- due to Hecke). Furthermore, the function $Q(2 s, a)$ also fulfills the following splendid property by Theorem 1.2:

-(0)- The function $\phi(s)$ has infinitely many zeros on the line $\sigma=1 / 4$.

Because Knopp did not explicitly provide solutions composed of zeta or $L$-functions that satisfy the conditions -(1)-, -(2)-, and -(3)-, we cannot see that the zeta or $L$-functions in his theorem fulfill any other noteworthy property. However, we show that the zeta function $Q(s, a)$ defined by (1.1) satisfies the functional equation (H2) in Theorem 1.1, or fulfills the condition -(2)- and some variations of -(1)- and -(3a)-, -(3b)-, or -(3)- in Corollaries 1.3, 1.4, and 1.5. Furthermore, we prove that the zeta function $Q(s, a)$ has $\gg T$ zeros on the segment $(1 / 2,1 / 2+i T)$ in Theorem 1.2. 


\section{Proofs}

2.1. Functional equation and integral representation. First, we prove Theorem 1.1, namely, the functional equation (1.2)

Proof of Theorem 1.1. For simplicity, we put

$$
Z(s, a):=\zeta(s, a)+\zeta(s, 1-a), \quad P(s, a):=\operatorname{Li}(s, a)+\operatorname{Li}(s, 1-a) .
$$

Note that one has $2 Q(s, a)=Z(s, a)+P(s, a)$. For $\sigma>1$, it is known that

$$
\zeta(1-s, a)=\frac{2 \Gamma(s)}{(2 \pi)^{s}}\left\{\cos \left(\frac{\pi s}{2}\right) \sum_{n=1}^{\infty} \frac{\cos 2 \pi n a}{n^{s}}+\sin \left(\frac{\pi s}{2}\right) \sum_{n=1}^{\infty} \frac{\sin 2 \pi n a}{n^{s}}\right\}
$$

(see for example [15, Theorem 2.3.1]). Thus, we have

$$
Z(1-s, a)=\frac{4 \Gamma(s)}{(2 \pi)^{s}} \cos \left(\frac{\pi s}{2}\right) \sum_{n=1}^{\infty} \frac{\cos 2 \pi n a}{n^{s}}=\frac{2 \Gamma(s)}{(2 \pi)^{s}} \cos \left(\frac{\pi s}{2}\right) P(s, a) .
$$

In addition, for $\sigma<0$, it holds that

$$
\operatorname{Li}(1-s, a)=\frac{\Gamma(s)}{(2 \pi)^{s}}\left(e^{\pi i s / 2} \zeta(s, a)+e^{-\pi i s / 2} \zeta(s, 1-a)\right), \quad 0<a<1
$$

from [1, Exercises 12.2 and 12.3]. Hence, one has

$$
P(1-s, a)=\frac{2 \Gamma(s)}{(2 \pi)^{s}}\left(\cos \left(\frac{\pi s}{2}\right) \zeta(s, a)+\cos \left(\frac{\pi s}{2}\right) \zeta(s, 1-a)\right)=\frac{2 \Gamma(s)}{(2 \pi)^{s}} \cos \left(\frac{\pi s}{2}\right) Z(s, a) .
$$

We note that the functions $\zeta(s, a)$ and $Z(s, a)$ are regular for all $s \in \mathbb{C}$ except $s=1$ (see [1, Chapter 12]) and the functions $\operatorname{Li}(s, a)$ and $P(s, a)$ with $0<a<1$ are analytically continuable to an entire function (see [15, Chapter 2.2]). Hence, the function $Q(s, a)$ is regular for all $s \in \mathbb{C}$ except $s=1$, where there is a simple pole with residue 1 . Therefore, by using the identity theorem and the functional equations of $Z(s, a)$ and $P(s, a)$ above, we have

$$
\begin{aligned}
& 2 Q(1-s, a)=Z(1-s, a)+P(1-s, a) \\
= & \frac{2 \Gamma(s)}{(2 \pi)^{s}} \cos \left(\frac{\pi s}{2}\right)(P(s, a)+Z(s, a))=\frac{2 \Gamma(s)}{(2 \pi)^{s}} \cos \left(\frac{\pi s}{2}\right) 2 Q(s, a)
\end{aligned}
$$

which implies the functional equation of $Q(s, a)$.

Next, we show the following integral representation of $\pi^{-s / 2} \Gamma(s / 2) Q(s, a)$.

Proposition 2.1. Define $G_{a}(u)$ by

$$
G_{a}(u):=\sum_{n \in \mathbb{Z}}\left(\exp \left(-\pi u^{2}(n+a)^{2}\right)+\exp \left(-\pi u^{2} n^{2}+i 2 \pi n a\right)\right) .
$$

Then, for $0<\Re(s)<1$, we have

$$
\pi^{-s / 2} \Gamma\left(\frac{s}{2}\right) Q(s, a)=\int_{0}^{\infty} u^{-s}\left(G_{a}(u)-1-\frac{1}{u}\right) d u .
$$


Proof. For $\Re(s)>1$, we have

$$
\begin{aligned}
& 2 \int_{0}^{\infty} u^{s-1} \sum_{n \in \mathbb{Z}} \exp \left(-\pi u^{2}(n+a)^{2}\right) d u \\
= & 2 \sum_{n=0}^{\infty} \int_{0}^{\infty} u^{s-1} e^{-\pi u^{2}(n+a)^{2}} d u+2 \sum_{n=0}^{\infty} \int_{0}^{\infty} u^{s-1} e^{-\pi u^{2}(n+1-a)^{2}} d u
\end{aligned}
$$

The first infinite series can be written as

$$
\sum_{n=0}^{\infty} \int_{0}^{\infty} e^{-v}\left(\frac{v / \pi}{(n+a)^{2}}\right)^{s / 2-1} \frac{d v / \pi}{(n+a)^{2}}=\pi^{-s / 2} \Gamma\left(\frac{s}{2}\right) \sum_{n=0}^{\infty}(n+a)^{-s} .
$$

Hence, we obtain

$$
2 \int_{0}^{\infty} u^{s-1} \sum_{n \in \mathbb{Z}} \exp \left(-\pi u^{2}(n+a)^{2}\right) d u=\pi^{-s / 2} \Gamma\left(\frac{s}{2}\right)(\zeta(s, a)+\zeta(s, 1-a)) .
$$

Similarly, when $\Re(s)>1$, one has

$$
\begin{aligned}
& 2 \int_{0}^{\infty} u^{s-1} \sum_{0 \neq n \in \mathbb{Z}} \exp \left(-\pi u^{2} n^{2}+i 2 \pi n a\right) d u \\
= & 2 \sum_{n=0}^{\infty} \int_{0}^{\infty} u^{s-1} e^{2 \pi i n a-\pi u^{2} n^{2}} d u+2 \sum_{n=0}^{\infty} \int_{0}^{\infty} u^{s-1} e^{2 \pi i n(1-a)-\pi u^{2} n^{2}} .
\end{aligned}
$$

The first infinite series can be expressed as

$$
\sum_{n=0}^{\infty} \int_{0}^{\infty} e^{2 \pi i n a} e^{-v}\left(\frac{v / \pi}{n^{2}}\right)^{s / 2-1} \frac{d v / \pi}{n^{2}}=\pi^{-s / 2} \Gamma\left(\frac{s}{2}\right) \sum_{n=0}^{\infty} \frac{e^{2 \pi i n a}}{n^{s}}
$$

Thus, it holds that

$$
2 \int_{0}^{\infty} u^{s-1} \sum_{0 \neq n \in \mathbb{Z}} \exp \left(-\pi u^{2} n^{2}+i 2 \pi n a\right) d u=\pi^{-s / 2} \Gamma\left(\frac{s}{2}\right)(\operatorname{Li}(s, a)+\operatorname{Li}(s, 1-a)) .
$$

Therefore, when $\Re(s)>1$, we have

$$
\pi^{-s / 2} \Gamma\left(\frac{s}{2}\right) Q(s, a)=\int_{0}^{\infty} u^{s-1}\left(G_{a}(u)-1\right) d u .
$$

For $a, u>0$, it is well-known that (see $[11$, p. 13, (6)])

$$
\begin{aligned}
& \sum_{n \in \mathbb{Z}} \exp \left(-\pi u^{2}(n+a)^{2}\right)=\frac{1}{u} \sum_{n \in \mathbb{Z}} \exp \left(-\pi u^{-2} n^{2}+i 2 \pi n a\right), \\
& \sum_{n \in \mathbb{Z}} \exp \left(-\pi u^{2} n^{2}+i 2 \pi n a\right)=\frac{1}{u} \sum_{n \in \mathbb{Z}} \exp \left(-\pi u^{-2}(n+a)^{2}\right) .
\end{aligned}
$$

Hence, we easily obtain

$$
G_{a}(u)=u^{-1} G_{a}\left(u^{-1}\right), \quad u>0 .
$$

By using the equation above and changing the variable $u \rightarrow v^{-1}$, we have

$$
\pi^{-s / 2} \Gamma\left(\frac{s}{2}\right) Q(s, a)=\int_{0}^{\infty} v^{-s}\left(G_{a}(v)-\frac{1}{v}\right) d v
$$


when $\Re(s)>1$. Hence, we have

$$
\begin{aligned}
& \pi^{-s / 2} \Gamma\left(\frac{s}{2}\right) Q(s, a)=\int_{0}^{1} v^{-s}\left(G_{a}(v)-\frac{1}{v}\right) d v+\int_{1}^{\infty} v^{-s}\left(G_{a}(v)-\frac{1}{v}\right) d v \\
& =\int_{0}^{1} v^{-s}\left(G_{a}(v)-\frac{1}{v}\right) d v+\int_{1}^{\infty} v^{-s}\left(G_{a}(v)-1-\frac{1}{v}\right) d v+\frac{1}{s-1} .
\end{aligned}
$$

Let $a_{\star}:=\min \{a, 1-a\}$. From the definition of $G_{a}(u)$ and $(2.1)$, it holds that

$$
G_{a}(u)=\frac{1}{u}\left(1+O\left(\exp \left(-a_{\star}^{2} u^{-2}\right)\right)\right), \quad u \rightarrow 0 .
$$

Let $\Re(s)>0$. By the definition of $G_{a}(u)$ and the estimation above, we have

$$
\begin{aligned}
& \int_{0}^{1} v^{-s}\left(G_{a}(v)-\frac{1}{v}\right) d v \ll \int_{0}^{1} v^{-\sigma-1} \exp \left(-a_{\star}^{2} v^{-2}\right) d v<\infty, \\
& \int_{1}^{\infty} v^{-s}\left(G_{a}(v)-1-\frac{1}{v}\right) d v \ll \int_{1}^{\infty} v^{-\sigma-1} d v+\int_{1}^{\infty}\left(G_{a}(v)-1\right) d v \\
& \ll \int_{1}^{\infty} \sum_{n=1}^{\infty} \exp \left(-\pi v n^{2}\right) d v<\infty .
\end{aligned}
$$

Hence, both integrals in $(2.2)$ converge when $\Re(s)>0$. Clearly, one has

$$
\frac{1}{s-1}=-\int_{0}^{1} v^{-s} d v
$$

for $\Re(s)<1$. Therefore, we obtain the integral representation in Proposition 2.1

2.2. Lemmas. We contrast the integrals $J_{a}(t)$ and $I_{a}(t)$ given by

$$
\begin{aligned}
J_{a}(t) & :=\frac{1}{2 \pi} \int_{t-k}^{t+k}\left|\pi^{-1 / 4-i u / 4} \Gamma\left(\frac{1+2 i u}{4}\right) Q(1 / 2+i u, a) e^{\pi u / 4} e^{-u \delta / 2}\right| d u \\
I_{a}(t) & :=\frac{e^{i \pi / 8} e^{-i \delta / 4}}{2 \pi} \int_{t-k}^{t+k} \pi^{-1 / 4-i u / 4} \Gamma\left(\frac{1+2 i u}{4}\right) Q(1 / 2+i u, a) e^{\pi u / 4} e^{-u \delta / 2} d u
\end{aligned}
$$

to show Theorem 1.2. This idea is parallel to the proof in [5, Chapter 11.2] or [22, Chapter 10.7]. Note that $\left|J_{a}(t)\right|>\left|I_{a}(t)\right|$ if the interval of integration of $I_{a}(t)$ contains roots of $Q(s, a)=0$ on $\Re(s)=1 / 2$ and $\left|J_{a}(t)\right|=\left|I_{a}(t)\right|$ otherwise. The key to the proof of Theorem 1.2 is to estimate the total length of all the integral intervals satisfying $\left|J_{a}(t)\right|>\left|I_{a}(t)\right|$. For this purpose, we present some lemmas.

Lemma 2.2. Let $a \neq 1 / 4, t$ and $k$ be sufficiently large. Then, we have

$$
\begin{aligned}
& \int_{t-k}^{t+k}|Q(1 / 2+i v, a)| d v \geq 2 k|\cos (2 \pi a)|-C_{1}(a)-\frac{C_{2}(a) k^{2}}{(t-k)^{1 / 2}} \\
& -\left|\sum_{a_{*}=a, 1-a} \sum_{2 \leq n<t / a_{\star}}\left(\frac{\sin \left(k \log \left(n+a_{*}\right)\right)}{\left(n+a_{*}\right)^{1 / 2+i t} \log \left(n+a_{*}\right)}+\frac{e^{2 \pi i a_{*} n} \sin (k \log n)}{n^{1 / 2+i t} \log n}\right)\right|,
\end{aligned}
$$

where $C_{1}(a)$ and $C_{2}(a)$ are some positive constants depend on a. 
Proof. For $a_{*}=a$ or $1-a$, the following approximate functional equations are shown (see [15, Theorems 3.1.3 and 3.1.2]). Suppose $2 \pi \leq|\tau| \leq \pi x$. Then, one has

$$
\zeta\left(1 / 2+i \tau, a_{*}\right)=\sum_{n=0}^{x} \frac{1}{\left(n+a_{*}\right)^{1 / 2+i \tau}}+O\left(x^{-1 / 2}\right) .
$$

Moreover, for $|t| \leq \pi a_{*} x$, it holds that

$$
\operatorname{Li}\left(1 / 2+i \tau, a_{*}\right)=\sum_{n=1}^{x} \frac{e^{2 \pi i a_{*} n}}{n^{1 / 2+i \tau}}+O\left(x^{-1 / 2}\right) .
$$

Let $a_{\star}:=\min \{a, 1-a\}$ again. Then, for $t-k \leq v \leq t+k$, we have

$$
\begin{aligned}
& \zeta\left(1 / 2+i v, a_{*}\right)=\sum_{0 \leq n<t / a_{\star}} \frac{1}{\left(n+a_{*}\right)^{1 / 2+i v}}+E_{\zeta}\left(v, a_{*}\right)+O\left(v^{-1 / 2}\right), \\
& \operatorname{Li}\left(1 / 2+i v, a_{*}\right)=\sum_{1 \leq n<t / a_{\star}} \frac{e^{2 \pi i a_{*} n}}{n^{1 / 2+i v}}+E_{\mathrm{Li}}\left(v, a_{*}\right)+O\left(v^{-1 / 2}\right),
\end{aligned}
$$

where $E_{\zeta}\left(v, a_{*}\right)$ is defined by

$$
E_{\zeta}\left(v, a_{*}\right):= \begin{cases}\sum_{t / a_{\star} \leq n<v / a_{\star}}\left(n+a_{*}\right)^{-1 / 2-i v} & t \leq v \\ -\sum_{v / a_{\star} \leq n<t / a_{\star}}\left(n+a_{*}\right)^{-1 / 2-i v} & t>v .\end{cases}
$$

The function $E_{\mathrm{Li}}\left(v, a_{*}\right)$ is defined similarly. Because $E_{\zeta}\left(v, a_{*}\right)$ consists of at most $2 k / a_{\star}$ terms each of modulus at most $\left(n+a_{*}\right)^{-1 / 2} \leq a_{\star}^{-1 / 2}(t-k)^{-1 / 2}$, we obtain that for $t-k \leq$ $v \leq t+k$,

$$
\Re\left(\sum_{0 \leq n<t / a_{\star}} \frac{1}{\left(n+a_{*}\right)^{1 / 2+i v}}-\zeta\left(1 / 2+i v, a_{*}\right)\right) \leq \frac{2 k a_{\star}^{-3 / 2}}{(t-k)^{1 / 2}}+O\left(v^{-1 / 2}\right) \leq \frac{C_{2}^{\prime}\left(a_{*}\right) k}{(t-k)^{1 / 2}}
$$

for some positive constant $C_{2}^{\prime}\left(a_{*}\right)$. Similarly, for some constant $C_{2}^{\prime \prime}\left(a_{*}\right)>0$, it holds that

$$
\Re\left(\sum_{1 \leq n<t / a_{\star}} \frac{e^{2 \pi i a_{*} n}}{n^{1 / 2+i v}}-\operatorname{Li}\left(1 / 2+i v, a_{*}\right)\right) \leq \frac{2 k a_{\star}^{-3 / 2}}{(t-k)^{1 / 2}}+O\left(v^{-1 / 2}\right) \leq \frac{C_{2}^{\prime \prime}\left(a_{*}\right) k}{(t-k)^{1 / 2}} .
$$

Assume $0<a<1 / 4$ and put $M(k, a):=4 k \cos (2 \pi a)-2 C_{1}(a)$ and

$$
M(k, t, a):=4 k \cos (2 \pi a)-2 C_{1}(a)-\frac{2 C_{2}(a) k^{2}}{(t-k)^{1 / 2}},
$$

where $0<C_{2}^{\prime}(a)+C_{2}^{\prime \prime}(a)+C_{2}^{\prime}(1-a)+C_{2}^{\prime \prime}(1-a) \leq C_{2}(a)$. For simplicity, we put $\sum_{*, \star}:=\sum_{a_{*}=a, 1-a} \sum_{2 \leq n<t / a_{\star}}$. Then, we have

$$
\begin{aligned}
& 2 \int_{t-k}^{t+k}|Q(1 / 2+i v, a)| d v \geq 2 \int_{t-k}^{t+k} \Re(Q(1 / 2+i v, a)) d v \\
\geq & M(k, a)+\int_{t-k}^{t+k}\left(\Re \sum_{*, \star}\left(\frac{1}{\left(n+a_{*}\right)^{1 / 2+i v}}+\frac{e^{2 \pi i a_{*} n}}{n^{1 / 2+i v}}\right)-\frac{C_{2}(a) k}{(t-k)^{1 / 2}}\right) d v \\
= & M(k, t, a)+\Re \sum_{*, \star}\left(\frac{2 \sin \left(k \log \left(n+a_{*}\right)\right)}{\left(n+a_{*}\right)^{1 / 2+i t} \log \left(n+a_{*}\right)}+\frac{2 e^{2 \pi i a_{*} n} \sin (k \log n)}{n^{1 / 2+i t} \log n}\right)
\end{aligned}
$$


which implies Lemma 2.2 with $0<a<1 / 4$. When $1 / 4<a<1 / 2$, based on the inequality

$$
\int_{t-k}^{t+k}|Q(1 / 2+i v, a)| d v \geq-\int_{t-k}^{t+k} \Re(Q(1 / 2+i v, a)) d v,
$$

we obtain Lemma 2.2 with $1 / 4<a<1 / 2$.

Lemma 2.3. Put $a_{\star}:=\min \{a, 1-a\}$ and $a_{*}=a$ or $1-a$. Let $A \leq t \leq B$ with $B \geq A \geq 2 / a_{\star}$. Then, it holds that

$$
\begin{aligned}
& \int_{A}^{B}\left|\sum_{2 \leq n<t / a_{\star}} \frac{\sin \left(k \log \left(n+a_{*}\right)\right)}{\left(n+a_{*}\right)^{1 / 2+i t} \log \left(n+a_{*}\right)}\right| d t \leq C_{3}\left(a_{*}\right) B, \\
& \int_{A}^{B}\left|\sum_{2 \leq n<t / a_{\star}} \frac{e^{2 \pi i n a_{*}} \sin (k \log n)}{n^{1 / 2+i t} \log n}\right| d t \leq C_{4}\left(a_{*}\right) B,
\end{aligned}
$$

for some positive constants $C_{3}\left(a_{*}\right)$ and $C_{4}\left(a_{*}\right)$.

Proof. First, we estimate the integral

$$
\begin{aligned}
& \int_{A}^{B}\left|\sum_{2 \leq n<t / a_{\star}} \frac{\sin \left(k \log \left(n+a_{*}\right)\right)}{\left(n+a_{*}\right)^{1 / 2+i t} \log \left(n+a_{*}\right)}\right|^{2} d t= \\
& \int_{A}^{B} \sum_{2 \leq n, m<t / a_{\star}} \frac{\sin \left(k \log \left(n+a_{*}\right)\right) \sin \left(k \log \left(m+a_{*}\right)\right)}{\left(n+a_{*}\right)^{1 / 2}\left(m+a_{*}\right)^{1 / 2} \log \left(n+a_{*}\right) \log \left(m+a_{*}\right)}\left(\frac{m+a_{*}}{n+a_{*}}\right)^{i t} d t .
\end{aligned}
$$

For the terms with $n=m$, we have

$$
\int_{A}^{B} \sum_{2 \leq n<t / a_{\star}} \frac{\sin ^{2}\left(k \log \left(n+a_{*}\right)\right)}{\left(n+a_{*}\right)\left(\log \left(n+a_{*}\right)\right)^{2}} d t \ll \int_{A}^{B} \sum_{2 \leq n<t / a_{\star}} \frac{1}{n(\log n)^{2}} d t .
$$

It should be noted that the infinite series $\sum_{n=2}^{\infty} n^{-1}(\log n)^{-2}$ converges. Each of the terms with $n \neq m$ is of the form

$$
\frac{\sin \left(k \log \left(n+a_{*}\right)\right) \sin \left(k \log \left(m+a_{*}\right)\right)}{\left(n+a_{*}\right)^{1 / 2}\left(m+a_{*}\right)^{1 / 2} \log \left(n+a_{*}\right) \log \left(m+a_{*}\right)} \int_{b}^{B}\left(\frac{m+a_{*}}{n+a_{*}}\right)^{i t} d t,
$$

where $b:=\max \left(A, a_{\star} m, a_{\star} n\right)$. Thus, regardless of the value of $b$, its absolute value is bounded above by

$$
\frac{2\left(n+a_{*}\right)^{-1 / 2}\left(m+a_{*}\right)^{-1 / 2}}{\log \left(n+a_{*}\right) \log \left(m+a_{*}\right)\left|\log \left(\left(n+a_{*}\right) /\left(m+a_{*}\right)\right)\right|} \ll \frac{n^{-1 / 2} m^{-1 / 2}}{\log n \log m|\log (n / m)|} .
$$

It is shown in $[5$, p. 236] that

$$
\sum_{2 \leq n \neq m \leq B} \frac{n^{-1 / 2} m^{-1 / 2}}{\log n \log m|\log (n / m)|} \ll B .
$$

Therefore, it holds that

$$
\int_{A}^{B}\left|\sum_{2 \leq n<t / a_{\star}} \frac{\sin \left(k \log \left(n+a_{*}\right)\right)}{\left(n+a_{*}\right)^{1 / 2+i t} \log \left(n+a_{*}\right)}\right|^{2} d t \ll B .
$$


From the inequality above and the the Cauchy-Schwarz inequality, we have

$$
\int_{A}^{B}\left|\sum_{2 \leq n<t / a_{\star}} \frac{\sin \left(k \log \left(n+a_{*}\right)\right)}{\left(n+a_{*}\right)^{1 / 2+i t} \log \left(n+a_{*}\right)}\right| d t \ll(B-A)^{1 / 2} B^{1 / 2} \ll B,
$$

which implies the first inequality of Lemma 2.3.

Second, consider the integral

$$
\begin{aligned}
& \int_{A}^{B}\left|\sum_{2 \leq n<t / a_{\star}} \frac{e^{2 \pi i n a_{*}} \sin (k \log n)}{n^{1 / 2+i t} \log n}\right|^{2} d t= \\
& \int_{A}^{B} \sum_{2 \leq n, m<t / a_{\star}} \frac{e^{2 \pi i n a_{*}} e^{-2 \pi i m a_{*}} \sin (k \log n) \sin (k \log m)}{n^{1 / 2} m^{1 / 2} \log n \log m}\left(\frac{m}{n}\right)^{i t} d t .
\end{aligned}
$$

Obviously, for the terms with $n=m$, we have

$$
\int_{A}^{B} \sum_{2 \leq n<t / a_{\star}} \frac{e^{2 \pi i n a_{*}} e^{-2 \pi i n a_{*}} \sin ^{2}(k \log n)}{n(\log n)^{2}} d t \leq \int_{A}^{B} \sum_{2 \leq n<t / a_{\star}} \frac{1}{n(\log n)^{2}} d t .
$$

For each of the terms with $n \neq m$, we have

$$
\left|\int_{b}^{B} \frac{e^{2 \pi i n a_{*}} e^{-2 \pi i m a_{*}} \sin (k \log n) \sin (k \log m)}{n^{1 / 2} m^{1 / 2} \log n \log m}\left(\frac{m}{n}\right)^{i t} d t\right| \ll \frac{n^{-1 / 2} m^{-1 / 2}}{\log n \log m|\log (n / m)|} .
$$

Thus, we have the second inequality of Lemma 2.3 from (2.4) and the Cauchy-Schwarz inequality.

2.3. Proof of the existence of zeros on the critical line. Let $k$ be a positive real number, $x$ be a complex number with $|x|=1$ and $|\Im(\log x)| \leq \pi / 4$, and put

$$
I_{x, k}(s, a):=\frac{1}{2 \pi i} \int_{s-i k}^{s+i k} \pi^{-v / 2} \Gamma\left(\frac{v}{2}\right) Q(v, a) x^{v-1} d v .
$$

Then, by Proposition 2.1, the function $I_{x, k}(s, a)$ can be expressed as

$$
\begin{aligned}
& \frac{1}{2 \pi i} \int_{s-i k}^{s+i k} \int_{0}^{\infty}\left(\frac{u}{x}\right)^{-v}\left(G_{a}(u)-1-\frac{1}{u}\right) \frac{d u d v}{x} \\
= & \frac{1}{2 \pi i} \int_{s-i k}^{s+i k} \int_{0}^{\infty / x}\left(G_{a}(x w)-1-\frac{1}{x w}\right) \frac{d w d v}{w^{v}} .
\end{aligned}
$$

By Cauchy's integral theorem and the fact that the function $G_{a}(u)-1$ approaches zero rapidly as $u$ tends to infinity along any ray $u=x w$ in the wedge $|\Im(\log x)| \leq \pi / 4, w \in \mathbb{R}$, the integral above is equal to

$$
\begin{aligned}
& \frac{1}{2 \pi i} \int_{s-i k}^{s+i k} \int_{0}^{\infty} w^{-v}\left(G_{a}(x w)-1-\frac{1}{x w}\right) d w d v \\
& =\int_{0}^{\infty}\left(\frac{1}{2 \pi i} \int_{s-i k}^{s+i k} w^{-v} d v\right)\left(G_{a}(x w)-1-\frac{1}{x w}\right) d w \\
& =\frac{1}{\pi} \int_{0}^{\infty} \frac{w^{-s} \sin (k \log w)}{\log w}\left(G_{a}(x w)-1-\frac{1}{x w}\right) d w
\end{aligned}
$$


This expresses $I_{x, k}(s, a)$ as the transform of an operator and shows, from the ParsevalPlancherel identity (see [5, p. 216, line 7]), that

$$
\frac{1}{2 \pi i} \int_{1 / 2-i \infty}^{1 / 2+i \infty}\left|I_{x, k}(s, a)\right|^{2} d s=\frac{1}{\pi^{2}} \int_{0}^{\infty}\left|\frac{\sin (k \log w)}{\log w}\right|^{2}\left|G_{a}(x w)-1-\frac{1}{x w}\right|^{2} d w .
$$

Note that under the change of variable $w \rightarrow w^{-1}$, the form $d w$ becomes $-d w / w^{2}$, the factor $\sin (k \log w) / \log w$ is unchanged, and the function $G_{a}(x w)-1-(x w)^{-1}$ becomes

$$
G_{a}\left(\frac{1}{\bar{x} w}\right)-1-\bar{x} w=\bar{x} w\left(\frac{1}{\bar{x} w} G_{a}\left(\frac{1}{\bar{x} w}\right)-\frac{1}{\bar{x} w}-1\right)=\bar{x} w\left(G_{a}(\bar{x} w)-1-\frac{1}{\bar{x} w}\right),
$$

where $x^{-1}=\bar{x}$, from (2.1). Thus, the integral of the right-hand side of (2.6) is equal to twice the integral from 1 to $\infty$. The first step is deriving an upper bound of the integral given by $(2.6)$.

Lemma 2.4. Let $I_{x, k}(s, a)$ be defined as in (2.5) with $x=e^{-i \pi / 4} e^{i \delta / 2}$. Then, there exists a constant $K>0$ such that given $\varepsilon>0$ the inequality

$$
\frac{1}{2 \pi i} \int_{1 / 2-i \infty}^{1 / 2+i \infty}\left|I_{x, k}(s, a)\right|^{2} d s<\frac{K k+\varepsilon k^{2}}{\delta^{1 / 2}}
$$

holds for all sufficiently small $\delta>0$ (with $k>0$ being arbitrary).

Proof. From the inequality

$$
\left|\frac{\sin k y}{y}\right| \leq \begin{cases}k & 0 \leq \pi / k \\ y^{-1} & y \geq \pi / k\end{cases}
$$

the integral of the left-hand side of (2.6) is bounded above by

$$
\frac{2 k^{2}}{\pi^{2}} \int_{1}^{e^{\pi / k}}\left|G_{a}(x w)-1-\frac{1}{x w}\right|^{2} d w+\frac{2}{\pi^{2}} \int_{e^{\pi / k}}^{\infty}\left|G_{a}(x w)-1-\frac{1}{x w}\right|^{2} \frac{d w}{(\log w)^{2}} .
$$

According to the inequality $|A+B|^{2} \leq 2|A|^{2}+2|B|^{2}$, where $A, B \in \mathbb{C}$, the first integral in $(2.7)$ is at most

$$
\frac{4 k^{2}}{\pi^{2}} \int_{1}^{e^{\pi / k}}\left|G_{a}(x w)-1\right|^{2} d w+\frac{4 k^{2}}{\pi^{2}} \int_{1}^{e^{\pi / k}} \frac{d w}{w^{2}} .
$$

Obviously, the second definite integral is $4 k^{2} \pi^{-2}\left(1-e^{-\pi / k}\right)$. From $|A+B|^{2} \leq 2|A|^{2}+2|B|^{2}$ again, the first integral is bounded above by

$$
\frac{8 k^{2}}{\pi^{2}} \int_{1}^{e^{\pi / k}}\left|\sum_{n \in \mathbb{Z}} \exp \left(-\pi x^{2} w^{2}(n+a)^{2}\right)\right|^{2} d w+\frac{8 k^{2}}{\pi^{2}} \int_{1}^{e^{\pi / k}}\left|\sum_{0 \neq n \in \mathbb{Z}} \exp \left(-\pi x^{2} w^{2} n^{2}+i 2 \pi n a\right)\right|^{2} d w
$$

Let $x=e^{i \pi / 4} e^{-i \delta / 2}$ so that $x^{2}=\sin \delta+i \cos \delta$. Then, the integrand in the first and second integrals respectively become

$$
\sum_{a_{b}, a_{\sharp}} \sum_{n, m=0}^{\infty} \exp \left(G_{n, m}^{a_{b}, a_{\sharp}}(w, \delta)\right), \quad \sum_{a_{b}, a_{\sharp}} \sum_{n, m=1}^{\infty} \exp \left(H_{n, m}^{a_{b}, a_{\sharp}}(w, \delta)\right),
$$


where the sum $\sum_{a_{b}, a_{\sharp}}$ taken over $\left(a_{b}, a_{\sharp}\right)=(a, a),(a, 1-a),(1-a, a),(1-a, 1-a)$ and the functions $G_{n, m}^{a_{b}, a_{\sharp}}(w, \delta)$ and $G_{n, m}^{a_{b}, a_{\sharp}}(w, \delta)$ are defined by

$$
\begin{aligned}
& G_{n, m}^{a_{b}, a_{\sharp}}(w, \delta):=-\pi\left(\left(n+a_{b}\right)^{2}+\left(m+a_{\sharp}\right)^{2}\right) w^{2} \sin \delta-i \pi\left(\left(n+a_{b}\right)^{2}-\left(m+a_{\sharp}\right)^{2}\right) w^{2} \cos \delta, \\
& H_{n, m}^{a_{b}, a_{\sharp}}(w, \delta):=-\pi\left(n^{2}+m^{2}\right) w^{2} \sin \delta-i \pi\left(n^{2}-m^{2}\right) w^{2} \cos \delta+i 2 \pi n a_{b}-i 2 \pi m a_{\sharp},
\end{aligned}
$$

respectively. We divide each double sum $\sum_{n, m=0}^{\infty}$ and $\sum_{n, m=1}^{\infty}$ into three sums, one in which $n=m$, one in which $n>m$ and one in which $n<m$ because both double sums in (2.8) converge absolutely. If $n=m$, we have

$$
\begin{aligned}
& \sum_{a_{b}, a_{\sharp}}\left(\sum_{n=0}^{\infty} \exp \left(G_{n, n}^{a_{b}, a_{\sharp}}(w, \delta)\right)+\sum_{n=1}^{\infty} \exp \left(H_{n, n}^{a_{b}, a_{\sharp}}(w, \delta)\right)\right) \\
& \leq C_{5}(a) \sum_{n=1}^{\infty} \exp \left(-2 \pi n^{2} w^{2} \sin \delta\right)
\end{aligned}
$$

for some positive constant $C_{5}(a)$. From the inequality

$$
\frac{8 k^{2}}{\pi^{2}} \int_{1}^{e^{\pi / k}} \sum_{n=1}^{\infty} \exp \left(-2 \pi n^{2} w^{2} \sin \delta\right) d w \ll \frac{k^{2}}{\pi^{2}} \int_{1}^{e^{\pi / k}} \frac{d w}{w(2 \sin \delta)^{1 / 2}} \ll k \delta^{-1 / 2},
$$

which is proved in $\left[5\right.$, p. 231 , line 7 from the bottom], for some positive constant $C_{6}(a)$, it holds that

$$
\begin{aligned}
& \frac{8 k^{2}}{\pi^{2}} \int_{1}^{e^{\pi / k}} \sum_{a_{b}, a_{\sharp}}\left(\sum_{n=0}^{\infty} \exp \left(G_{n, n}^{a_{b}, a_{\sharp}}(w, \delta)\right)+\sum_{n=1}^{\infty} \exp \left(H_{n, n}^{a_{b}, a_{\sharp}}(w, \delta)\right)\right) d w \\
& \leq C_{6}(a) k \delta^{-1 / 2} .
\end{aligned}
$$

Next, we will estimate the definite integral of the remaining terms $n \neq m$ of (2.8) from 1 to $e^{\pi / k}$. The terms with $m>n$ are the complex conjugates of those with $m<n$, so it suffices to estimate the latter. Consider the integral

$$
\frac{8 k^{2}}{\pi^{2}} \int_{1}^{e^{\pi / k}} \exp \left(-\pi\left((n+a)^{2}+(m+a)^{2}\right) w^{2} \sin \delta-i \pi\left((n+a)^{2}-(m+a)^{2}\right) w^{2} \cos \delta\right) d w
$$

when $n>m$. The real part of the integral is

$$
\begin{gathered}
\frac{8 k^{2}}{\pi^{2}} \int_{1}^{e^{\pi / k}} f(w, a) \cos V(w, a) d w, \\
\text { where } \quad\left\{\begin{array}{l}
f(w, a):=\exp \left(-\pi\left((n+a)^{2}+(m+a)^{2}\right) w^{2} \sin \delta\right), \\
V(w, a):=\pi\left((n+a)^{2}-(m+a)^{2}\right) w^{2} \cos \delta .
\end{array}\right.
\end{gathered}
$$

Because $\cos \delta$ is positive for a small $\delta>0, V(w, a)$ is a monotone increasing function with respect to $w$, and this integral can be rewritten in terms of the variable $V$ as

$$
\frac{8 k^{2}}{\pi^{2}} \int_{V(1)}^{V\left(e^{\pi / k}\right)} \frac{f}{V^{\prime}} \cos V d V
$$


where $f$ and $V^{\prime}$ are functions of $V$ by composition with the inverse function $V \rightarrow w$. By [5, Lemma in p. 197] and the fact that $f$ is decreasing and $V^{\prime}$ is increasing, the integral above is not more than

$$
\frac{8 k^{2}}{\pi^{2}} \frac{2 f(1)}{V^{\prime}(1)}=\frac{16 k^{2}}{\pi^{2}} \frac{\exp \left(-\pi\left((n+a)^{2}+(m+a)^{2}\right) w^{2} \sin \delta\right)}{2 \pi\left((n+a)^{2}-(m+a)^{2}\right) \cos \delta} .
$$

A similar estimate can be applied to the imaginary part and to both the real and imaginary parts of the following integral

$$
\frac{8 k^{2}}{\pi^{2}} \int_{1}^{e^{\pi / k}} \exp \left(-\pi\left(n^{2}+m^{2}\right) w^{2} \sin \delta-i \pi\left(n^{2}-m^{2}\right) w^{2} \cos \delta+i 2 \pi n a-i 2 \pi m a\right) d w .
$$

Hence, for some positive constant $C_{7}(a)$ and $a_{b}, a_{\sharp}=a$ or $1-a$, we have

$$
\begin{aligned}
& \frac{8 k^{2}}{\pi^{2}}\left|\int_{1}^{e^{\pi / k}} \exp \left(G_{n, m}^{a_{b}, a_{\sharp}}(w, \delta)\right) d w\right|, \quad \frac{8 k^{2}}{\pi^{2}}\left|\int_{1}^{e^{\pi / k}} \exp \left(H_{n, m}^{a_{b}, a_{\sharp}}(w, \delta)\right) d w\right| \\
& \leq C_{7}(a) k^{2} \frac{\exp \left(-\pi\left(n^{2}+m^{2}\right) \sin \delta\right)}{\left(n^{2}-m^{2}\right) \cos \delta} .
\end{aligned}
$$

For any $\varepsilon>0$ and for all sufficiently small $\delta>0$, we have

$$
\sum_{n=1}^{\infty} \sum_{m=0}^{n-1} \frac{k^{2} \exp \left(-\pi\left(n^{2}+m^{2}\right) \sin \delta\right)}{\left(n^{2}-m^{2}\right)} \ll k^{2} \sum_{n=1}^{\infty} \exp \left(-n^{2} \sin \delta\right) \frac{\log n}{n} \leq \varepsilon k^{2} \delta^{-1 / 2} .
$$

from [5, p. 233, line 1]. Therefore, it holds that

$$
\frac{8 k^{2}}{\pi^{2}} \int_{1}^{e^{\pi / k}} \sum_{a_{b}, a_{\sharp}} \sum_{n=1}^{\infty}\left(\sum_{m=0}^{n-1} \exp \left(G_{n, m}^{a_{b}, a_{\sharp}}(w, \delta)\right)+\sum_{m=1}^{n-1} \exp \left(H_{n, m}^{a_{b}, a_{\sharp}}(w, \delta)\right)\right) d w \leq \varepsilon k^{2} \delta^{-1 / 2} .
$$

Similar methods prove that the same estimation applies to the second integral in (2.7). We can easily see that

$$
\frac{2}{\pi^{2}} \int_{e^{\pi / k}}^{\infty}(\log w)^{-2}\left|\frac{1}{x w}\right|^{2} d w \leq \frac{2}{\pi^{2}}\left(\log e^{\pi / k}\right)^{-2} \int_{e^{\pi / k}}^{\infty} \frac{d w}{w^{2}} \leq \varepsilon k^{2} \delta^{-1 / 2} .
$$

It is shown in [5, p. 233, line 15] that

$$
\int_{e^{\pi / k}}^{\infty}(\log w)^{-2} \sum_{n=1}^{\infty} \exp \left(-2 \pi n^{2} w^{2} \sin \delta\right) \ll k \delta^{-1 / 2} .
$$

Hence, from (2.9), it holds that

$$
\int_{e^{\pi / k}}^{\infty}(\log w)^{-2} \sum_{a_{b}, a_{\sharp}}\left(\sum_{n=0}^{\infty} \exp \left(G_{n, n}^{a_{b}, a_{\sharp}}(w, \delta)\right)+\sum_{n=1}^{\infty} \exp \left(H_{n, n}^{a_{b}, a_{\sharp}}(w, \delta)\right)\right) d w \ll k \delta^{-1 / 2} .
$$

Moreover, it is shown in [5, p. 233, line 12 from the bottom] that

$$
\begin{aligned}
& \int_{e^{\pi / k}}^{\infty} \sum_{n=1}^{\infty} \sum_{m<n} \exp \left(-\pi\left(n^{2}+m^{2}\right) w^{2} \sin \delta-i \pi\left(n^{2}-m^{2}\right) w^{2} \cos \delta\right) \frac{d w}{(\log w)^{2}} \\
& \ll \sum_{n=1}^{\infty} \sum_{m<n} \frac{\exp \left(-\pi\left(n^{2}+m^{2}\right) \sin \delta\right)}{\left(\log e^{\pi / k}\right)^{2}\left(n^{2}-m^{2}\right)} \leq \varepsilon k^{2} \delta^{-1 / 2} .
\end{aligned}
$$


Thus, by the inequality above and modifying the proof of (2.10), we have

$$
\sum_{a_{b}, a_{\sharp}} \sum_{n=1}^{\infty} \int_{e^{\pi / k}}^{\infty}\left(\sum_{m=0}^{n-1} \exp \left(G_{n, m}^{a_{b}, a_{\sharp}}(w, \delta)\right)+\sum_{m=1}^{n-1} \exp \left(H_{n, m}^{a_{b}, a_{\sharp}}(w, \delta)\right)\right) \frac{d w}{(\log w)^{2}} \leq \varepsilon k^{2} \delta^{-1 / 2} .
$$

The estimate of the integral (2.6) is unchanged under $e^{i \pi / 4} e^{-i \delta / 2} \rightarrow e^{-i \pi / 4} e^{i \delta / 2}$ by the complex conjugate of $I_{x, k}(s, a)$. Hence, Lemma 2.4 is obtained.

Next, we estimate the integral

$$
J_{k}(t, a):=\frac{1}{2 \pi} \int_{t-k}^{t+k}\left|\pi^{-1 / 4-i u / 4} \Gamma\left(\frac{1+2 i u}{4}\right) Q(1 / 2+i u, a) e^{\pi u / 4} e^{-u \delta / 2}\right| d u .
$$

When $x=e^{-i \pi / 4} e^{i \delta / 2}$, we have

$$
\begin{aligned}
& I_{x, k}(1 / 2+i t, a)=\frac{1}{2 \pi} \int_{t-k}^{t+k} \pi^{-1 / 4-i u / 4} \Gamma\left(\frac{1+2 i u}{4}\right) Q(1 / 2+i u, a) x^{-1 / 2} x^{i u} d u \\
& =\frac{x^{-1 / 2}}{2 \pi} \int_{t-k}^{t+k} \pi^{-1 / 4-i u / 4} \Gamma\left(\frac{1+2 i u}{4}\right) Q(1 / 2+i u, a) e^{\pi u / 4} e^{-u \delta / 2} d u .
\end{aligned}
$$

For simplicity, we put

$$
I_{a}(t):=I_{x, k}(1 / 2+i t, a), \quad J_{a}(t):=J_{k}(t, a) .
$$

Then, we have $J_{a}(t) \geq\left|I_{a}(t)\right|$ for all $t \in \mathbb{R}$ and $J_{a}(t)=\left|I_{a}(t)\right|$ whenever the interval of integration of $I_{a}(t)$ contains no roots of $Q(s, a)=0$ on the line $\Re(s)=1 / 2$. The basic idea of the proof is to show that in a suitable sense $J_{a}(t)$ is much larger than $\left|I_{a}(t)\right|$ on average. Thus, estimates of $J_{a}(t)$ from below are required. Stirling's formula yields

$$
\begin{aligned}
\Gamma\left(\frac{1+2 i u}{4}\right)|Q(1 / 2+i u, a)| e^{-\pi u / 4} & \gg u^{3 / 4} e^{-u \pi / 4} u^{-1}|Q(1 / 2+i u, a)| e^{\pi u / 4} \\
& \gg u^{-1 / 4}|Q(1 / 2+i u, a)|
\end{aligned}
$$

when $u$ is sufficiently large. Thus, we have the following:

Lemma 2.5. For sufficiently large $t>0$, it holds that

$$
J_{a}(t) \gg(t+k)^{-1 / 4} e^{-(t+k) \delta / 2} \int_{t-k}^{t+k}|Q(1 / 2+i u, a)| d u .
$$

Now, we are in a position to prove the main theorem. Note that the proof below is based on the argument in [5, Chapter 11.2] (see also [22, Chapter 10.7]). When $a=1 / 4$ or $1 / 2$, it holds that

$$
Q(s, 1 / 2)=\left(2^{s}+2^{1-s}-2\right) \zeta(s), \quad 2 Q(s, 1 / 4)=\left(2^{2 s}-2^{s}+2^{2-2 s}-2^{1-s}\right) \zeta(s)
$$

by (1.6) (see also [17, Section 2.2]). Therefore, we suppose $0<a<1 / 4$ or $1 / 4<a<1 / 2$ which implies that $\cos (2 \pi a) \neq 0$ (see Lemma 2.2 ).

Proof of Theorem 1.2. Let $\nu$ be the number of zeros of $Q(1 / 2+i t, a)$ in the interval $\{0 \leq t \leq B+k\}$. And let the line $\Re(s)=1 / 2$ be divided into intervals of length $k$ and for each of the $\nu$ zeros strike out the interval which contains it and the intervals which adjoin this one. Let $S$ be the subset of $\{A \leq t \leq B\}$ consisting of points which do not lie in the stricken intervals. Then, the total length of the intervals of $S$ is not less than $B-A-3 \nu k$ because a length of at most $3 k$ was stricken for each zero. Note that $\left|I_{a}(t)\right|=J_{a}(t)$ for 
all $t \in S$. Put $I:=\int_{S}\left|I_{a}(t)\right| d t$. Then, by Lemma 2.5 and the fact that there are no zeros between $t-k$ and $t+k$, we have

$$
I=\int_{S} J_{a}(t) d t \gg \int_{S}(B+k)^{-1 / 4} e^{-(B+k) \delta / 2} \int_{t-k}^{t+k}|Q(1 / 2+i u, a)| d u d t .
$$

From Lemmas 2.2 and 2.3, we have

$$
\begin{aligned}
& I \gg(B+k)^{-1 / 4} e^{-(B+k) \delta / 2} \int_{S}\left(2 k \cos |(2 \pi a)|-C_{1}(a)-\frac{C_{2}(a) k^{2}}{(t-k)^{1 / 2}}\right. \\
&\left.-\left|\sum_{a_{*}=a, 1-a} \sum_{2 \leq n<t / a_{\star}}\left(\frac{\sin \left(k \log \left(n+a_{*}\right)\right)}{\left(n+a_{*}\right)^{1 / 2+i t} \log \left(n+a_{*}\right)}+\frac{e^{2 \pi i a_{*} n} \sin (k \log n)}{n^{1 / 2+i t} \log n}\right)\right|\right) d t \\
& \gg(B+k)^{-1 / 4} e^{-(B+k) \delta / 2}\left(2 k(B-A-3 \nu k)|\cos (2 \pi a)|-C_{7}(a) B-C_{8}(a) k^{2} B^{1 / 2}\right)
\end{aligned}
$$

for some positive constants $C_{7}(a)$ and $C_{8}(a)$. Let $(B+k) \delta=2$, which can be regarded as a choice of $B$ given $\delta$ and $k$, and let $B-A=\delta^{-1}$ which can be regarded as a choice of $A$. Then, the estimation above becomes

$$
\begin{aligned}
I & \gg \delta^{1 / 4}\left(2 k\left(\delta^{-1}-3 \nu k\right)|\cos (2 \pi a)|-2 C_{7}(a) \delta^{-1}-\sqrt{2} C_{8}(a) k^{2} \delta^{-1 / 2}\right) \\
& \gg K_{1} k \delta^{-3 / 4}-K_{2} k^{2} \delta^{1 / 4} \nu-K_{3} \delta^{-3 / 4}-K_{4} k^{2} \delta^{-1 / 4},
\end{aligned}
$$

where $K_{1}, K_{2}, K_{3}$ and $K_{4}$ are positive constants (depending on $a$ ). In contrast, from Lemma 2.4, we have

$$
\begin{aligned}
I & \leq \int_{A}^{B}\left|I_{a}(t)\right| d t \leq\left(\int_{A}^{B} 1^{2} d t\right)^{1 / 2}\left(\int_{A}^{B}\left|I_{a}(t)\right|^{2} d t\right)^{1 / 2} \\
& \leq\left.\left. B^{1 / 2}\left|\int_{1 / 2-i \infty}^{1 / 2+i \infty}\right| I_{x, k}(s, a)\right|^{2} d s\right|^{1 / 2}=K_{5} \frac{\left(K k+\varepsilon k^{2}\right)^{1 / 2}}{\delta^{3 / 4}} .
\end{aligned}
$$

Therefore, it holds that

$$
K_{1} k \delta^{-3 / 4}-K_{2} k^{2} \delta^{1 / 4} \nu-K_{3} \delta^{-3 / 4}-K_{4} k^{2} \delta^{-1 / 4} \leq K_{5} \delta^{-3 / 4}\left(K k+\varepsilon k^{2}\right)^{1 / 2},
$$

which is equivalent to

$$
\nu \geq \frac{K_{1}}{K_{2}} k^{-1} \delta^{-1}-\frac{K_{3}}{K_{2}} k^{-2} \delta^{-1}-\frac{K_{4}}{K_{2}} \delta^{-1 / 2}-\frac{K_{5}}{K_{2}} k^{-1} \delta^{-1}\left(\frac{K}{k}+\varepsilon\right)^{1 / 2} .
$$

We can make the coefficient of $k^{-1} \delta^{-1}$ on the right-hand side positive by choosing $\varepsilon>0$ and $k^{-1}>0$ to be sufficiently small. Hence, with this fixed $k>0$, it has been shown that for all sufficiently small $\delta>0$, the number of roots on the line segment from $1 / 2$ to $1 / 2+i 2 \delta^{-1}$ is greater than $K_{6} \delta^{-1}-K_{7} \delta^{-1 / 2}$ with $K_{6}>0$.

Acknowledgments. The author would like to thank Professors Kohji Matsumoto and Masatoshi Suzuki for their useful advice. The author would like to also thank the referee for a careful reading of the manuscript and valuable comments and remarks. The author was partially supported by JSPS, grant no. 16K05077. 


\section{REFERENCES}

[1] T. M. Apostol, Introduction to Analytic Number Theory. Undergraduate Texts in Mathematics, Springer, New York, 1976.

[2] S. Baier, K. Srinivas and U. K. Sangale, A note on the gaps between zeros of Epstein's zeta-functions on the critical line. Funct. Approx. Comment. Math. 57 (2017), no. 2, 235-253.

[3] E. Bombieri and D. A. Hejhal, On the distribution of zeros of linear combinations of Euler products. Duke Math. J. 80 (1995), no. 3, 821-862.

[4] J. B. Conrey, More than two fifths of the zeros of the Riemann zeta function are on the critical line. J. Reine Angew. Math. 399 (1989), 1-26.

[5] H. M. Edwards, Riemann's zeta function. Reprint of the 1974 original [Academic Press, New York]. Dover Publications, Inc., Mineola, NY, 2001.

[6] P. Epstein Zur Theorie allgemeiner Zetafunktionen. II. Math. Ann. 63 (1906), no. 2, 205-216

[7] S. M. Gonek, The zeros of Hurwitz's zeta function on $\sigma=1 / 2$. Analytic number theory (Philadelphia, Pa., 1980), pp. 129-140, Lecture Notes in Math., 899, Springer, Berlin-New York, 1981.

[8] H. Hamburger, Über die Riemannsche Funktionalgleichung der $\zeta$-Funktion. (German) Math. Z. 10 (1921), no. 3-4, 240-254.

[9] G. H. Hardy and J. E. Littlewood, The zeros of Riemann's zeta-function on the critical line. Math. Z. 10 (1921), no. 3-4, 283-317.

[10] E. Hecke, Herleitung des Euler-Produktes der Zetafunktion und einiger L-Reihen aus ihrer Funktionalgleichung. Math. Ann. 119 (1944). 266-287.

[11] A. A. Karatsuba and S. M. Voronin, The Riemann zeta-function. Translated from the Russian by Neal Koblitz. De Gruyter Expositions in Mathematics, 5. Walter de Gruyter \& Co., Berlin, 1992.

[12] M. Knopp, On Dirichlet series satisfying Riemann's functional equation. Invent. Math. 117 (1994), no. 3, 361-372.

[13] P. Kühn, N. Robles and D. Zeindler, On a mollifier of the perturbed Riemann zeta-function. J. Number Theory 174, (2017), 274-321.

[14] M. Jutila and K. Srinivas, Gaps between the zeros of Epstein's zeta-functions on the critical line. Bull. London Math. Soc. 37 (2005), no. 1, 45-53.

[15] A. Laurinčikas and R. Garunkštis, The Lerch zeta-function. Kluwer Academic Publishers, Dordrecht, 2002.

[16] N. Levinson, More than one third of the zeros of Riemann's zeta function are on $\sigma=1 / 2$. Adv. Math.13 (1974), 383-436.

[17] T. Nakamura, On the real and complex zeros of the quadrilateral zeta function, preprint, arXiv:2001.01981.

[18] T. Nakamura, The values of zeta functions composed by the Hurwitz and periodic zeta functions at integers, preprint, arXiv:2006.03300.

[19] H. S. Potter and E. C. Titchmarsh, The Zeros of Epstein's Zeta-Functions. Proc. London Math. Soc. (2) 39 (1935), no. 5, 372-384.

[20] A. Sankaranarayanan, Zeros of quadratic zeta-functions on the critical line. Acta Arith. 69 (1995), no. $1,21-38$.

[21] C. Siegel, Bemerkung zu einem Satz von Hamburger uber die Funktionalgleichung der Riemannschen Zetafunktion. Math. Ann. 86 (1922), no. 3-4, 276-279.

$[22]$ E. C. Titchmarsh, The theory of the Riemann zeta-function, Second edition. Edited and with a preface by D. R. Heath-Brown. The Clarendon Press, Oxford University Press, New York, 1986.

(T. Nakamura) Department of Liberal Arts, Faculty of Science and Technology, Tokyo University of Science, 2641 Yamazaki, NodA-Shi, Chiba-Ken, 278-8510, Japan

Email address: nakamuratakashi@rs.tus.ac.jp

$U R L:$ https://sites.google.com/site/takashinakamurazeta/ 